\title{
Improving the consistency of multi-LOD CityGML datasets by removing redundancy
}

\author{
Filip Biljecki * \\ Section GIS Technology, Delft University of Technology, The Netherlands \\ Hugo Ledoux \\ Section GIS Technology, Delft University of Technology, The Netherlands \\ Jantien Stoter \\ Section GIS Technology, Delft University of Technology, The Netherlands \\ Kadaster, Product and Process Innovation, Apeldoorn, The Netherlands \\ Geonovum, Amersfoort, The Netherlands
}

\section{ORCID}

FB: http://orcid.org/0000-0002-6229-7749

HL: http://orcid.org/0000-0002-1251-8654

JS: http://orcid.org/0000-0002-1393-7279

* Corresponding author at f.biljecki@tudelft.nl

This is an Accepted Manuscript of an article published by Springer in the Lecture Notes in Geoinformation and Cartography. 3D Geoinformation Science: The Selected Papers of the 3D GeoInfo 2014 in 2015. The final publication is available at Springer via

http://doi.org/10.1007/978-3-319-12181-9_1

Cite as:

Biljecki, F., Ledoux, H., Stoter, J. (2015): Improving the Consistency of Multi-LOD CityGML Datasets by Removing Redundancy. Lecture Notes in Geoinformation and Cartography. 3D Geoinformation Science: The Selected Papers of the 3D GeoInfo 2014, Springer International Publishing, pp. 1-17. 


\begin{abstract}
The CityGML standard enables the modelling of some topological relationships, and the representation in multiple levels of detail (LODs). However, both concepts are rarely utilised in reality. In this paper we investigate the linking of corresponding geometric features across multiple representations. We describe the possible topological cases, show how to detect these relationships, and how to store them explicitly. A software prototype has been implemented to detect matching features within and across LODs, and to automatically link them by establishing explicit topological relationships (with XLink). The experiments ran on our test datasets show a considerable number of matched geometries. Further, this method doubles as a lossless data compression method, considering that the storage footprint in the consolidated datasets has been reduced from their dissociated counterparts.
\end{abstract}

Keywords: Multi-LOD; Topology; XLink; CityGML; Compression

\title{
1 Introduction
}

The OGC standard CityGML [17,31], and other 3D modelling standards such as COLLADA [23, 34 ] and ISO's X3D [22] allow the storage of multiple level of detail (LOD) representations of a model, in order to facilitate the multi-scale use of the models and to improve the computational efficiency of spatial operations [7].

Although 3D GIS datasets may contain multiple LODs, multi-LOD datasets are almost nonexistent in practice, and they are seldom linked beyond an administrative link between object identifiers (i.e. they share the same building ID) [8]. In our opinion, this situation is caused by the following deficiencies: (1) lack of consistency, i.e. there is redundancy in the acquisitionmodelling-storage process; (2) when using multi-LOD datasets, it is not always clear when and how to switch between LODs as it is the case in computer graphics; and (3) 3D generalisation specifications and implementations are not fully developed, limiting the generation of LODs other than the one being primarily constructed from the acquired dataset.

In this paper we focus on the first shortcoming by investigating the possible improvements in the consistency and storage of multi-LOD datasets, with a theory that is applicable also to singleLOD representations. It is our experience that in practice, besides exemplary models, single-LOD datasets do not contain the explicit representation of topological relationships, hence developing a joint method that is beneficial for both possibilities is important. We observe and take advantage of the practical fact that many of the stored geometries (primarily polygons) in 3D datasets are geometrically equal both within a single LOD and across multiple LODs. By determining the topological relationships between such reoccurring geometries and storing them explicitly, the consistency of 3D models can be increased, as we show in this paper. However, while developing the method, we have realised that in practice most of the geometries that are reoccurring are not identical and cannot be readily matched. Therefore, we have investigated other cases and covered them as well. 
This paper is focused towards CityGML and its LOD concept, however, most of the developed work is applicable to other formats. Our work consists of the following contributions: (1) we have investigated and described several cases of reoccurring geometries and introduce a terminology to distinguish them; (2) we have developed robust algorithms that efficiently index the geometries in CityGML datasets and that take advantage of the geometries that reoccur by explicitly storing their topological relationships; (3) we have developed a software prototype that analyses CityGML data and automatically computes explicit topological links between matching geometric features through the XML Linking Language (XLink) mechanism; and (4) we show with experiments that a considerable subset of data can be matched. We have tested the method on a synthetic dataset that contains buildings in LOD1, LOD2, and two variants of LOD3.

Because matched geometries are stored only once, the consolidated dataset is compressed without loss of information.

In Sec. 2 we explain the advantages of establishing explicit topological representations between geometric features. In Sec. 3 we describe possible topological cases, introduce our terminology, and present the algorithms that index, match, and consolidate the geometry of 3D datasets, primarily CityGML. The consolidation of the data is a technical challenge because it is done on a hierarchical data structure, and there might not be an optimal algorithm that is suited for all CityGML datasets.

The implementation and the results, presented in Sec. 4, show that after linking a higher degree of the consistency of the data is achieved, contributing to an efficient storage and maintenance. For instance, if the geometry of a feature is altered in one LOD, thanks to the established explicit topological representations this change may propagate through other LODs.

\section{Background and related work}

Consistency of 3D city models is an important topic in GIS, in which topology plays a prominent role $[15,28]$. Current research efforts focus on the relationships of features within the same representation, e.g. the validation of solids [27], and making use of topological data structuring to improve rendering performance of 3D city models on mobile devices [12]. To the extent of our knowledge, there is no related work to detect and link geometric features across multiple representations.

While this paper generally describes a way how to increase the consistency and to compute topological relationships in a model, it is focused on the maintenance and storage of (3D) GIS datasets, which is a topical subject in academia and industry $[2,36,37]$. Updates of models often introduce errors [18], so increasing consistency is one of the prerequisites for an efficient workflow.

In this section we describe the redundancy and benefits of an established topology with respect to the scalability of the models: for single representations (single-LOD datasets), and for multiple representations (multi-LOD datasets). 


\subsection{Single-LOD datasets}

Research that has been done in this topic is focused on the relationship of real-world features within the same representation $[14,16]$. For instance, the topology of two coinciding polygons, such as a wall shared by two buildings. The consistency that is achieved by establishing explicit topological relationships in practice simplifies the maintenance of the data and reduces the redundancy in the storage.

Fig. 1 shows an example of the benefit with respect to the maintenance of a 3D model. The left model (Fig. 1(a)) shows a building with a wall that contains a window. The polygon representing the wall is shown in red, and it contains a hole (inner ring), which is filled by another polygon representing the window. The interior ring of the wall polygon corresponds to the exterior ring of the polygon representing the window. In a model without established explicit topological relationships, the two features are not linked in any way. When the geometry of a part of the object is updated, e.g. the window is enlarged, the change does not affect the related geometry (i.e. hole of the wall), leading to redundancy in the process (see Fig. 1(b)). In a model with established topological representations, the change properly propagates to the related features (see Fig. 1(c) for the desirable outcome).

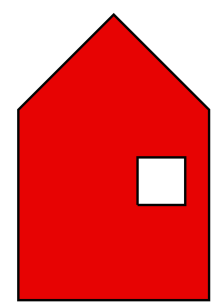

(a) Model of a building before the update (enlargement of the window).

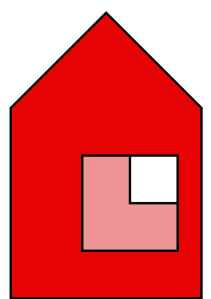

(b) Updated model without links resulting in inconsistency.

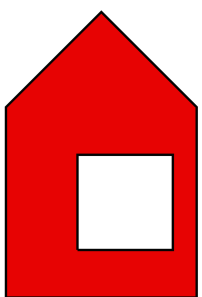

(c) Updated model with established links (desired case).

Figure 1: The determined explicit topological relationships in a dataset has a significant benefit to its maintenance. This example shows the benefit on a wall with a window that is being enlarged.

\subsection{Multi-LOD datasets}

On top of the redundancy in a single representation, that multiplies with each new representation in a multi-LOD dataset, there is also additional redundancy. For instance, should a feature be changed, in practice the update must be done manually for each representation. Further, because of the increasing complexity of the models, the size of the datasets substantially increases with the increase of the LOD, making the storage less feasible. The surge in the size is not only caused by 
the growth of the amount of details, but also because of the redundancy that could be removed, as we show later in the paper.

Therefore, despite the fact that this option is available in CityGML, models are usually derived in a single LOD representation. While multi-LOD datasets are rare, when they are available they are usually produced by generalisation from finer LODs (e.g. see $[1,4,40]$ ), for instance, as a bounding box of an LOD2 [11], or of an LOD3 including features such as antennas on roofs [30]. This is beneficial for this research, since it results in datasets where many of the geometries are preserved, and are identical in more than one representation. For instance, the ground surface of a building (i.e. GroundSurface) is usually identical in all representations.

Detecting and linking such occurrences would be a first step towards complementing the discussed practical shortcomings.

\section{Methodology}

We have developed a method that searches for matching geometries in the datasets and links them. Fig. 2 shows the desirable outcome of the algorithms with an example of three LODs where some of the geometries are reoccurring and are consequently linked.
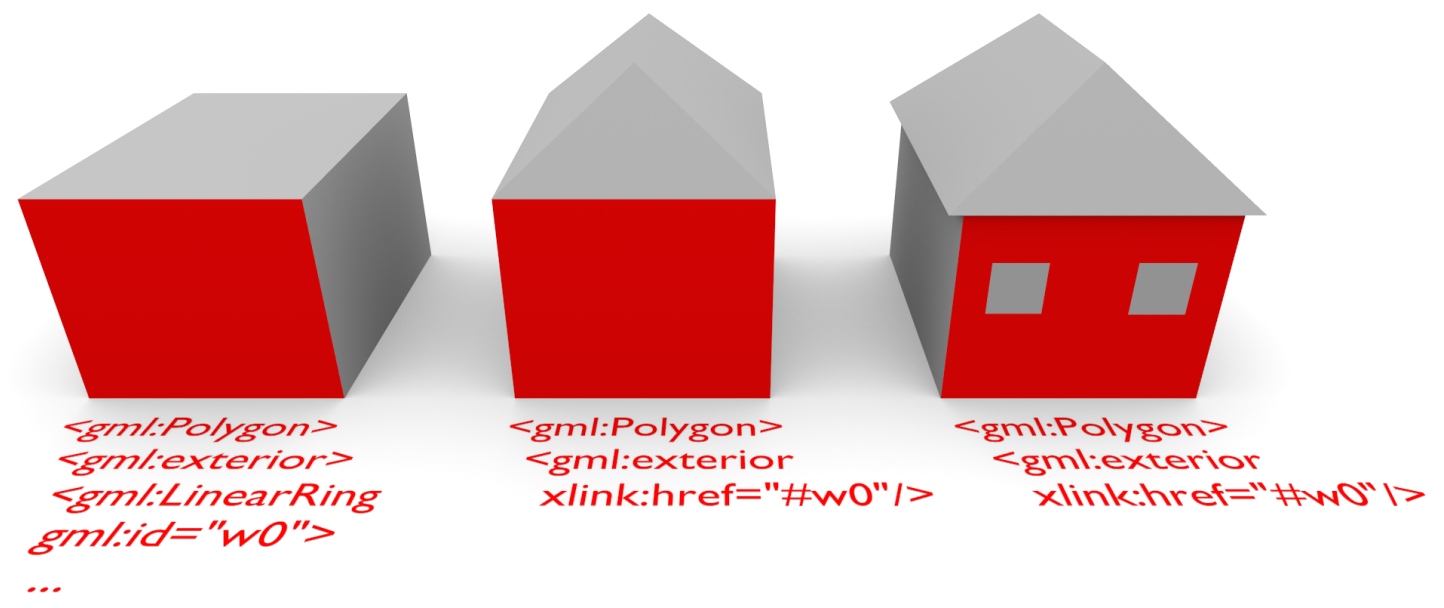

Figure 2: The rationale of the method. If two or more geometries are found to correspond, links are created. In this case two polygons (walls) are identical in two LODs, however, in one LOD the polygon has a hole, hence, only their exterior rings are linked. How and where to establish the links while balancing the maximisation of links and the topological structure is the main concern of the consolidation process.

While examining multi-LOD datasets we have realised that there are different cases of corresponding geometry, not only polygons that are identical and that can be directly referenced. For instance, polygons that share the exterior ring, but their interior is different (common in CityGML 
LOD3 where openings are allowed, see Fig. 2). Further, specific cases such as two equal polygons whose starting point is different should also be handled.

\subsection{Terminology}

In this paper we focus on the two geometric feature types: polygons and linear rings. We consider two or more geometric primitives identical if they are topologically and geometrically equivalent, i.e. they can be readily linked and re-used. The geometric representations of the ground plane of a building in two LODs are usually identical. Two or more primitives are partially identical if they are not identical and if their relation has one or more of the following properties which prevent them to be identical:

- The orientation of their vertices is different, i.e. their normals are reversed. For instance, two buildings share the same wall.

- They constitute different aspects of their parent primitive, e.g. two linear rings correspond, but one forms the exterior ring of its parent polygon, while the other forms the interior ring to describe a hole. A prominent example of this case is a wall with a hole that represents an opening-window or door which is stored separately.

- The number of points in their rings is not equal while the shape and location are identical. This is caused by redundant points $p_{i}$, where $p_{i-1}, p_{i}, p_{i+1}$ are collinear. The removal of such points would not compromise the shape and location of the polygon.

- The starting point in the linear ring is different. This discrepancy might be easily detected and corrected on-the-fly, hence it will not be particularly emphasised in the continuation of the paper.

Two geometries match if they are either identical or partially identical. When a match of two or more geometries is found, one is selected as the resource, and the rest are linked to it.

\subsection{Topological relationships}

In this section we show the possible cases of the matching geometry, i.e. topological relationships between polygons, and their constituting components-exterior and interior ring(s). We have investigated the possible cases, and their occurrences in real-world datasets, which we show in Tab. 1 . The sign $=$ denotes identical primitives, - partially identical, $\neq$ non-matching, and $\varnothing$ denotes no geometry. The matched primitives in each case are shown in red. In the example column, when two objects are separated, the example refers to the multi-LOD case.

While more "permutations" are possible, they are not valid according to GML, hence the list does not take into account invalid cases. One such example are two exterior rings that are identical, but their interior rings have a reversed orientation (which is shown in the last line as an exceptional example). 
Table 1: Cases of topological relationships of rings and polygons. The curved arrows denote the ring's orientation, while the long horizontal arrows indicate that there is a relation between one polygon's exterior to another polygon's interior.

\begin{tabular}{|c|c|c|c|c|c|}
\hline \multirow[t]{2}{*}{ Case } & \multicolumn{2}{|c|}{ Ring } & \multirow[t]{2}{*}{ Polygon } & \multirow[t]{2}{*}{ Graphical explanation } & \multirow[t]{2}{*}{ Real-world example } \\
\hline & Exterior & Interior & & & \\
\hline 0 & $\neq$ & $\neq$ & $\neq$ & $\sqrt{\square}$ & \\
\hline 1 & $=$ & $\varnothing$ & $=$ & $\sqrt{7}$ & \\
\hline 2 & $=$ & $=$ & $=$ & & \\
\hline 3 & $=$ & $\neq$ & $\neq$ & $\vec{\square}$ & \\
\hline 4 & - & $\varnothing$ & - & $f$ & \\
\hline 5 & - & - & - & 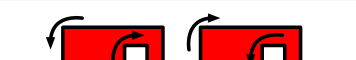 & \\
\hline 6 & - & $\neq$ & $\neq$ & $\sqrt{\square}$ & Unidentified \\
\hline 7 & $\neq$ & $=$ & $\neq$ & $\sqrt{6}$ & Unidentified \\
\hline 8 & $\neq$ & - & $\neq$ & $\sqrt{6}$ & \\
\hline 9 & $\longleftarrow$ & $\rightarrow$ & $\neq$ & $\sqrt{5}$ & Unidentified \\
\hline 10 & $\longleftarrow$ & $\rightarrow$ & $\neq$ & $\sqrt{\Gamma}$ & \\
\hline Invalid & $=$ & - & $?$ & $\sqrt{6} \sqrt{\square}$ & Not possible \\
\hline
\end{tabular}


The case 0 is the (usual) case where two primitives have no topological relation in the context of this research. Identical features rarely occur within the same LOD (in contrast to partially identical), so the cases 1 and 2 where the geometries of two features are identical are often present in multi-LOD datasets. Case 3 is also typical in multi-LOD datasets (again, see Fig. 2), while case 4 is more common in single-LOD datasets where two buildings share the same wall. The fifth case extends the previous with holes. Cases 6 and 7 are unusual in the real-world, and case 8 is similar to the fifth case in the occasion when the polygon is not identical. Cases 9 and 10 are cases of interchangeable roles of the exterior and interior rings, and might be rather considered as extended cases. Case 9 is uncommon, and case 10 is usually occurring in finer single-LOD datasets as the relation between a wall and an opening.

\subsection{Overview of the method}

The workflow of the method to match regards both matching primitives within the same LOD and across multiple LODs:

1. Indexing. Index all points, linear rings, and polygons in all LODs for efficiency (Sec. 3.4).

2. Matching. Detect matching geometries and flag them. Because of different topological relations, which have been introduced in the previous section, the detection of the matching geometries is done in multiple phases (Sec. 3.5).

3. Consolidation. Analyse the matched geometry and remove redundant data by replacing them with a link to one other matching representation resource, with modifications if necessary (Sec. 3.6).

Fig. 3 shows the simplified workflow of the method and the relation between the features when searching for matches. Because all features are indexed, the searching algorithm has a considerably reduced subset of potential matches. After ruling out non-matching features in the indexed subset, the algorithms detect the matched features and classify them according to the cases presented in the previous section.

The developed algorithm and the implemented software prototype are focused towards ideal cases where the vertices of the geometry are identical across LODs and where the geometries fully correspond. This is useful for datasets produced with generalisation, however, when used on datasets with a different lineage it might not produce results to the same extent. This could be solved by introducing the snapping of the points according to a tolerance threshold, and more sophisticated matching of similar geometries. The automatic matching of the representations that are acquired with different techniques would require employing more advanced algorithms such as $[3,41]$, extending related work done in cartography (e.g. $[10,39])$ to 3D GIS, and would probably result in a lossy compression (partial data discarding). 


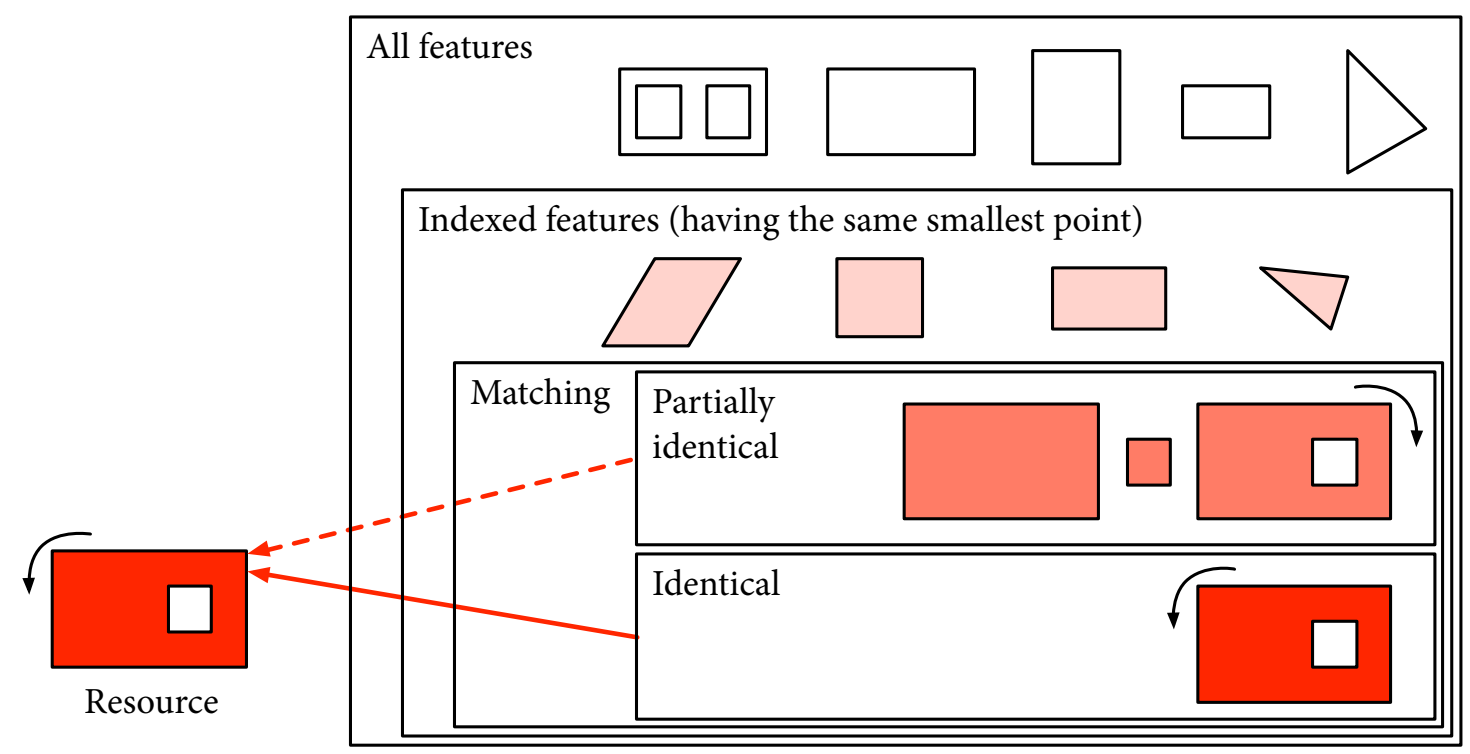

Figure 3: Simplified classification of the relationships and workflow of the method. The primitives in the dataset are first indexed, and then tested for matches, of which there are different categories.

\subsection{Algorithm for indexing the geometry}

In order to make the search for the matching geometry more efficient and enable the consolidation of larger datasets, the polygons and their rings are first indexed. We have decided to build an index where each polygon's ring is indexed according to its smallest point. The smallest point is the point with the smallest coordinate value, i.e. the one that is closest to $(-\infty,-\infty,-\infty)$. Each valid ring has one such point, hence, it can serve for the purpose of indexing. This point should not be confused with the starting point of the ring, which is not relevant here.

This step considerably reduces the search time for matches since in practice only a few nonmatching polygons share the same smallest point. As an example, Fig. 4 shows two different polygons that share the same smallest point. The comparison of such polygons for discarding the different geometries is described in the next step.

\subsection{Algorithm for the detection of matching geometries}

After indexing, the rings (both exterior and interior) are queried for their relations. The algorithm first removes vertices that are redundant (i.e. being collinear with its preceding and succeeding points). The algorithm is given in Alg. 1 . 


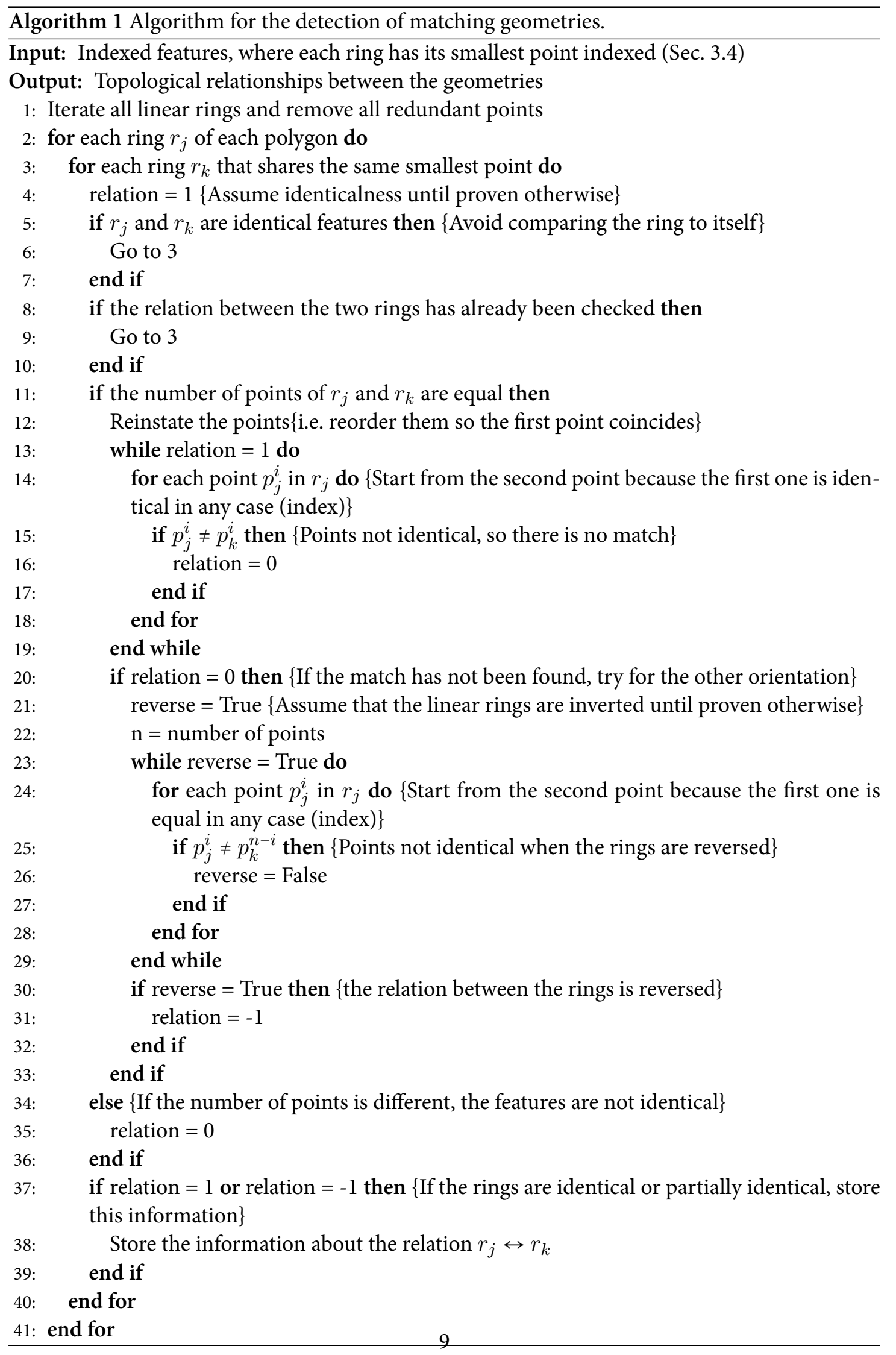




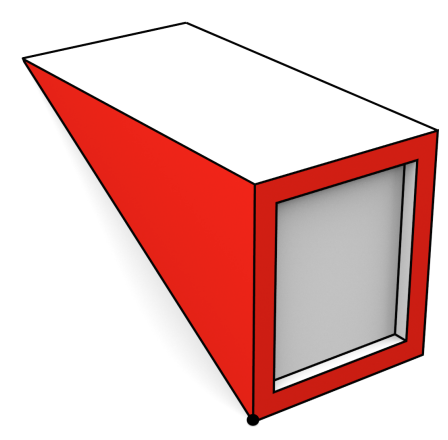

Figure 4: Multiple different polygons (shown here in red) may have the same smallest point (shown in black). In this case the two polygons are part of a dormer of a building.

\subsection{Algorithm for the consolidation of the data}

After detecting matching geometries, the last phase of the method involves consolidating the data (linking), i.e. analysing the relationships and determining the level of the relationship that can be linked. A straightforward solution would be to directly link the matching rings. However, because of the different cases and hierarchies, this phase is not forthright, and it can be solved in multiple ways. For instance, if two rings that form the exterior of two polygons match, this does not necessarily mean that the polygons can be matched right away, since the interior may be different (e.g. see case 3 in Tab. 1). Further, one of these rings may be an interior of another polygon, that is further related to another polygon in another way. Therefore, maximising the number of links that are established is the main concern when designing such algorithm, and cannot be solved simply by determining the frequency of the occurrences and selecting the topmost ring. Further, the development of the algorithm is associated with the content of a targeted dataset, as an algorithm might not be equally beneficial when employed for consolidating two different datasets. This problem is related to the field of data compression [20,33].

We have designed a top-down approach that first iterates polygons with holes, comparing the matched rings, and builds a hierarchy of features, continuing to polygons without holes. This is particularly beneficial for cases $1,2,3$ and 10 , which are the most common. The algorithm is given in Alg. 2.

\section{Implementation and results}

\subsection{Test data}

Because multi-LOD datasets are rare in practice, there is a difficulty to find input material for the testing of the prototype. The publicly available CityGML datasets that contain more than one LOD representation are limited to one or a few buildings (e.g. [19]). 


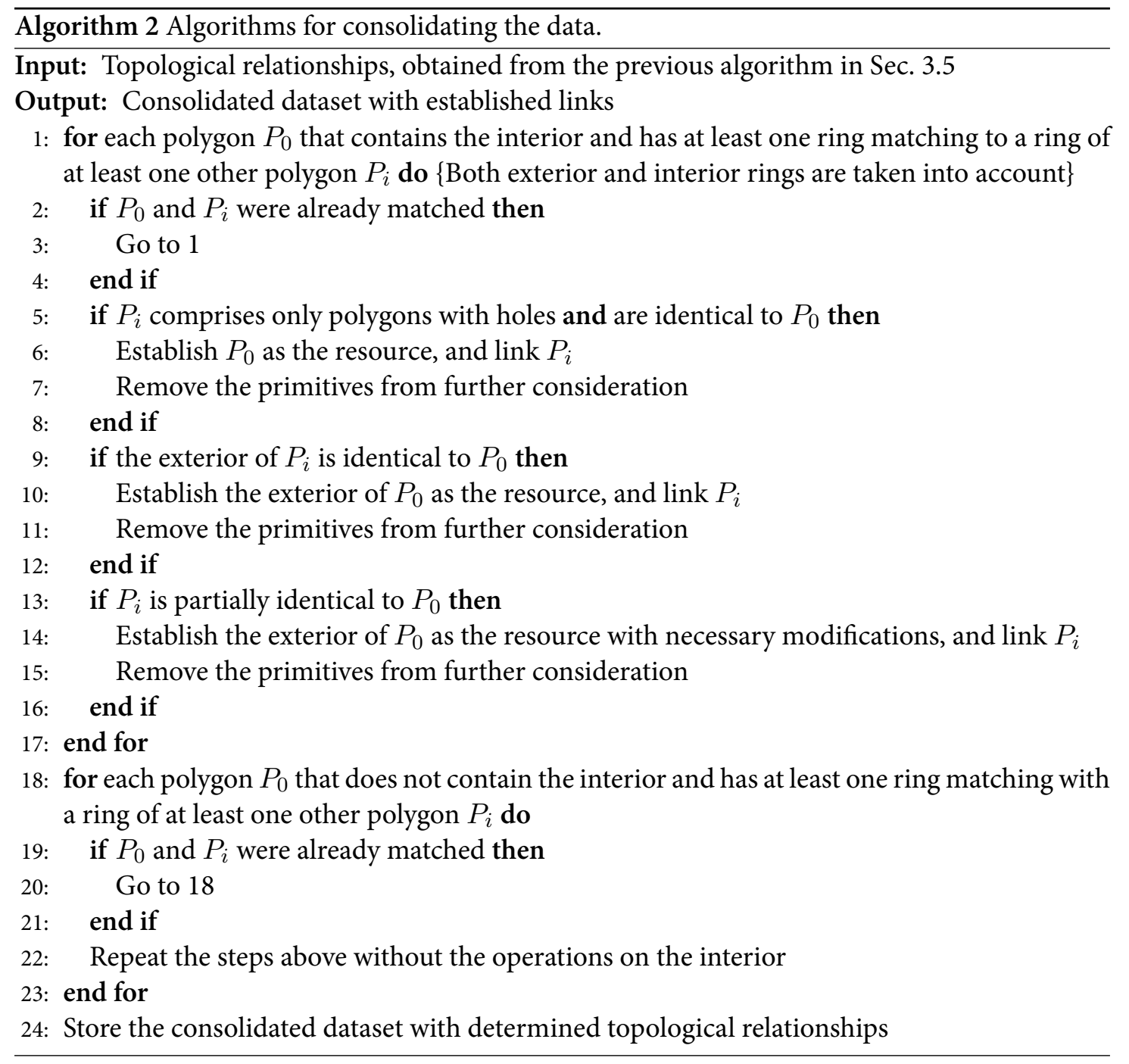


We have used a dataset in multiple LODs that was automatically generated from a parametrised description by the engine "Random3Dcity", which was presented in [6]. The dataset contains 100 buildings. Some of the LODs are represented in two ways, as a $<\mathrm{gml}$ : Solid $>$ or as semantically structured surfaces ( $\langle\mathrm{gml}$ : boundedBy $\rangle$ ). This is done in order to extend the experiments by comparing the different variations of the models that are valid [5, 29]. Further, two variants of LOD3 are available, in order to take into account the different levels of complexity that may occur in the same LOD range (see [7] for further details). The dataset contains six representations of each building, that are shown in Fig. 5 and described below:

LOD1s The standard LOD1 block model, represented as a $\langle\mathrm{gml}$ : Solid $>$. The top of the block model represents the height of the building at the eaves. The footprint represents the real footprint of the building ( $c f$. cadastral records).

LOD2s A model with simple standardised roof shapes. The footprint is the same as in LOD1. It is also stored as a solid.

LOD2b The semantically enriched boundary representation from which the previous model was generated.

LOD3s A solid obtained from the architecturaly detailed model. In comparison to the LOD2, it includes dormers and other objects that contribute to the internal volume of the building.

LOD3b A detailed model with openings (doors and windows). From the geometric perspective, the major difference with respect to the previous representations is that the polygons contain <gml: interior $>$.

LOD3+ A very detailed variant of LOD3 that contains smaller details such as embrasures of windows and doors (see Fig. 4 for example). Such models are rare in CityGML. This representation is important because it presents a considerable increase in the storage footprint comparing to the coarser representations, e.g. roughly twice the size of LOD3b.

Since CityGML does not support the simultaneous representation of more than the five standard LODs, the datasets have been stored in separate files.
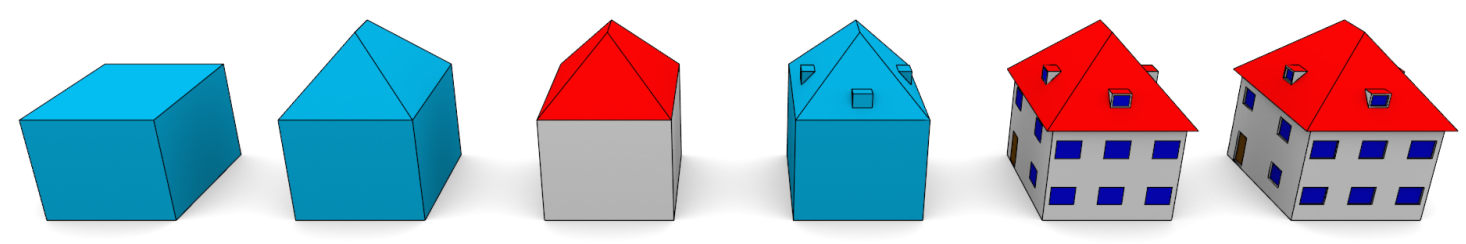

Figure 5: Visualisation of the six representations that are available in the test data. Their order from the left is the same as in the description in the text. 


\subsection{Implementation}

The implementation was done in Python. The XML Linking Language (XLink) mechanism was used to realise the links between the features. It allows elements to be inserted into Extensible Markup Language (XML) documents for creating and describing links between resources [38].

XLink has been used already in research projects that employ CityGML [25, 35, 24]. It is also mentioned in the GML standard [32,26] and in the CityGML standard [31] as the preferred way of explicitly storing the topological relationships. In CityGML, it is primarily used for referencing mutual geometries of two objects (e.g. a building and a building part), and for the re-use of the geometry in the semantically enriched boundary representation for the construction of solids [13].

The example below shows the code for a resource and a link. When two or more matching geometries are detected, the prototype adds an identifier to the gml:id attribute of the resource, i.e. the preserved single instance of the multiple matches. For the unambiguous identification of resources and links, the Universally Unique Identifier (UUID) standard has been used [21].

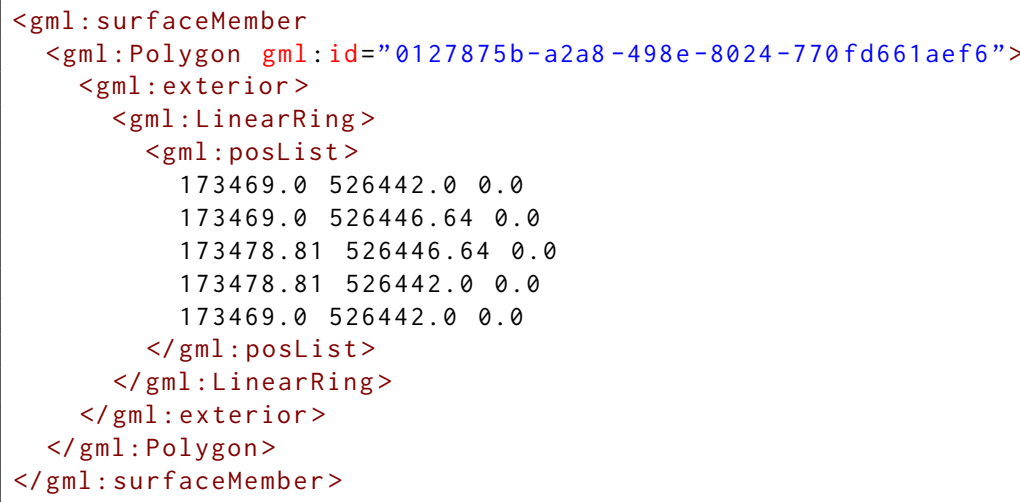

Afterwards, the contents of each matching geometry is removed and a link is added pointing to the resource which contains the geometry:

<gml: surfaceMember xlink:href="\#0127875b-a2a8-498e-8024-770fd661aef6"/>

Geometries that have opposite normals (reversed ring orientation) can be referenced with $<$ gml:OrientableSurface $>$. For instance, the reversed match of the polygon above may be stored as:

<gml:Orientablesurface orientation="-">

<gml: baseSurface xlink: href="\#0127875b-a2a8-498e-8024-770fd661aef6"/>

$</$ gml: Orientablesurface $>$

For reversing the linear rings, $<\mathrm{gml}$ : Or ientableCurve $>$ can be used.

These examples not only show the achieved consistency, but also the decrease in the storage footprint. 


\subsection{Results}

The implemented software prototype was run on the test dataset that contains diverse cases of matching geometries. After running, the results show considerable improvements in the consistency of the dataset. A significant number of polygons was found to match, both within singleLOD and multi-LOD data. Further, examining the results in details shown that some of the rings reoccured as many as 9 times, which reinforces the importance of linking such geometries.

The total number of polygons in the datasets is 13021 (of which $8 \%$ has interior) with 17029 rings. Indexing reduced the amount of queries by $99.88 \%$. Most of the points in the index referred to less than 10 rings (i.e. the number of rings that share the same smallest point), and within each comparison, an average of $42 \%$ of rings were found to match. The geometries were linked, and the consolidated data has been stored.

A useful insight in the established links is that all interior rings in the dataset are found to be matched to an exterior ring of another polygon. This is due to openings such as windows and doors, and closure surfaces.

Obviously, the obtained results such as the number of matched primitives (88\%), strongly depend on the used dataset, however, they give a good impression of the quantity of reoccurring geometries, for which there is no reason to store them more than once.

While the consolidation algorithm provides a good balance between simplicity and the end result (amount of consolidated data), its computational complexity is exponential, rendering it less feasible for larger datasets.

From the storage perspective, after the consolidation the size of the dataset was reduced by $20 \%$. Due to the highly repetitive structure of an XML schema such as CityGML, we do not expect that the consolidation of recurring geometries can further contribute to the reduction of the storage footprint. Further data compression of multi-LOD datasets should be sought in methods such as XML clustering [9].

\section{Conclusions and future work}

In this paper we have presented a method to analyse multi-LOD CityGML datasets by detecting matching geometries, and to automatically adapt them by storing the explicit representations of their topological relationships. This enhancement considerably improves the consistency of the model, leading to more efficient maintenance and storage. The software prototype that we have implemented shows significant improvements in that respect.

As a foundation of the work, we have developed a theoretical framework that describes cases of topological relationships occurring in reality, and we investigate more closely the XLink mechanism that provides the explicit modelling of some topological relationships in (City)GML. The work can be used for both within a representation and across multiple representations, doubling 
its purpose. Since our implementation is automatic, we hope that these improvements will contribute to the increased creation of multi-LOD datasets and their deeper integration.

While the tests involved only buildings, the presented algorithms are intended to work for other thematic classes as well. However, because of the lack of such datasets, testing possibilities are limited, and had to be done on a synthetic dataset produced by our random engine [6].

The matching algorithm is suited for ideal cases such as generalised datasets which contain identical primitives across LODs, and may fall short in datasets constructed with different acquisition techniques. Improvements in this direction are one of the aims that we plan for future work. Further, we plan to improve the algorithm that consolidates the data and to offer a few alternatives with different advantages and suitability.

\section{Acknowledgements}

We would like to thank our colleague Ken Arroyo Ohori for his suggestions, and the anonymous reviewers for their thorough comments which have helped us to improve the manuscript. This research is supported by the Dutch Technology Foundation STW, which is part of the Netherlands Organisation for Scientific Research (NWO), and which is partly funded by the Ministry of Economic Affairs. (Project code: 11300)

\section{References}

[1] Akmalia, R., Setan, H., Majid, Z., Suwardhi, D., Chong, A.: TLS for generating multi-LOD of 3D building model. IOP Conference Series: Earth and Environmental Science. Proceedings of the 8th International Symposium of the Digital Earth (ISDE8) 18, 1-8 (2014)

[2] Aringer, K., Roschlaub, R.: Bavarian 3D Building Model and Update Concept Based on LiDAR, Image Matching and Cadastre Information. In: Innovations in 3D Geo-Information Sciences, pp. 143-157. Springer International Publishing (2014)

[3] Arkin, E.M., Chew, L.P., Huttenlocher, D.P., Kedem, K., Mitchell, J.S.B.: An efficiently computable metric for comparing polygonal shapes. IEEE Transactions on Pattern Analysis and Machine Intelligence 13(3), 209-216 (1991)

[4] Baig, S.U., Abdul-Rahman, A.: Generalization of buildings within the framework of CityGML. Geo-spatial Information Science 16(4), 247-255 (2013)

[5] Benner, J., Geiger, A., Gröger, G., Häfele, K.H., Löwner, M.O.: Enhanced LOD concepts for virtual 3D city models. In: U. Isikdag (ed.) ISPRS Annals of the Photogrammetry, Remote Sensing and Spatial Information Sciences. Proceedings of the ISPRS 8th 3D GeoInfo Conference \& WG II/2 Workshop, pp. 51-61. Istanbul, Turkey (2013) 
[6] Biljecki, F., Ledoux, H., Stoter, J.: Error propagation in the computation of volumes in 3D city models with the Monte Carlo method. In: ISPRS Annals of the Photogrammetry, Remote Sensing and Spatial Information Sciences. Proceedings of the ISPRS/IGU Joint International Conference on Geospatial Theory, Processing, Modelling and Applications. Toronto, Canada (2014)

[7] Biljecki, F., Ledoux, H., Stoter, J., Zhao, J.: Formalisation of the level of detail in 3D city modelling. Computers, Environment and Urban Systems 48, 1-15 (2014)

[8] Biljecki, F., Zhao, J., Stoter, J., Ledoux, H.: Revisiting the concept of level of detail in 3D city modelling. In: U. Isikdag (ed.) ISPRS Annals of the Photogrammetry, Remote Sensing and Spatial Information Sciences. Proceedings of the ISPRS 8th 3D GeoInfo Conference \& WG II/2 Workshop, pp. 63-74. Istanbul, Turkey (2013)

[9] Dalamagas, T., Cheng, T., Winkel, K.J., Sellis, T.: Clustering XML Documents by Structure. In: G.A. Vouros, T. Panayiotopoulos (eds.) Lecture Notes in Computer Science. Methods and Applications of Artificial Intelligence, pp. 112-121. Springer Berlin Heidelberg (2004)

[10] Dilo, A., van Oosterom, P., Hofman, A.: Constrained tGAP for generalization between scales: The case of Dutch topographic data. Computers, Environment and Urban Systems 33(5), 388-402 (2009)

[11] El-Mekawy, M., Östman, A., Shahzad, K.: Towards interoperating CityGML and IFC building models: a unified model based approach. In: T.H. Kolbe, G. König, C. Nagel (eds.) Proceedings of the 5th International 3D GeoInfo Conference. Advances in 3D Geo-Information Sciences. Lecture Notes in Geoinformation and Cartography, pp. 73-93. Springer (2011)

[12] Ellul, C., Altenbuchner, J.: Investigating approaches to improving rendering performance of 3D city models on mobile devices. Geo-spatial Information Science 17(2), 73-84 (2014)

[13] Gröger, G., Coors, V.: Modeling Guide for 3D Objects. Tech. rep., SIG3D (2011)

[14] Gröger, G., Plümer, L.: How to Get 3-D for the Price of 2-D? Topology and Consistency of 3-D Urban GIS. GeoInformatica 9(2), 139-158 (2005)

[15] Gröger, G., Plümer, L.: How to achieve consistency for 3D city models. GeoInformatica 15(1), 137-165 (2009)

[16] Gröger, G., Plümer, L.: Topology of surfaces modelling bridges and tunnels in 3D-GIS. Computers, Environment and Urban Systems 35(3), 208-216 (2011)

[17] Gröger, G., Plümer, L.: CityGML - Interoperable semantic 3D city models. ISPRS Journal of Photogrammetry and Remote Sensing 71, 12-33 (2012)

[18] Gröger, G., Plümer, L.: Transaction rules for updating surfaces in 3D GIS. ISPRS Journal of Photogrammetry and Remote Sensing 69, 134-145 (2012)

[19] Häfele, K.H.: CityGML Model of the FJK-Haus. Institut für Angewandte Informatik (IAI), Karlsruher Institut für Technologie (2011) 
[20] Huffman, D.: A Method for the Construction of Minimum-Redundancy Codes. Proceedings of the IRE 40(9), 1098-1101 (1952)

[21] ISO: ISO/IEC 9834-8:2008(E) - Information technology - Open Systems Interconnection - Procedures for the operation of OSI Registration Authorities: Generation and registration of Universally Unique Identifiers (UUIDs) and their use as ASN.1 Object Identifier components (2008)

[22] ISO: ISO/IEC 19775-1:2013 Information technology - Computer graphics, image processing and environmental data representation - Extensible 3D (X3D) - Part 1: Architecture and base components (2013)

[23] ISO/TC 184: ISO/PAS 17506:2012 - Industrial automation systems and integration - COLLADA digital asset schema specification for 3D visualization of industrial data

[24] Iwaszczuk, D., Stilla, U.: A concept for assignment of textures to partially occluded faces of 3D city models stored in CityGML. In: T.H. Kolbe, G. König, C. Nagel (eds.) International Archives of Photogrammetry, Remote Sensing and Spatial Information Sciences. Proceedings of the 5th International 3D GeoInfo Conference, pp. 57-62. Berlin, Germany (2010)

[25] Kolbe, T.H.: Representing and exchanging 3D city models with CityGML. In: S. Zlatanova, L. Jiyeong (eds.) 3D Geo-Information Sciences, pp. 15-31. Springer Berlin Heidelberg (2009)

[26] Lake, R., Burggraf, D., Trninić, M., Rae, L.: Geography mark-up language (GML). Foundation for the Geo-web. John Wiley \& Sons, Inc. (2004)

[27] Ledoux, H.: On the Validation of Solids Represented with the International Standards for Geographic Information. Computer-Aided Civil and Infrastructure Engineering 28(9), 693-706 (2013)

[28] Ledoux, H., Meijers, M.: Topologically consistent 3D city models obtained by extrusion. International Journal of Geographical Information Science 25(4), 557-574 (2011)

[29] Löwner, M.O., Benner, J., Gröger, G., Häfele, K.H.: New Concepts for Structuring 3D City Models - an Extended Level of Detail Concept for CityGML Buildings. In: B. Murgante, S. Misra, M. Carlini, C.M. Torre, H.Q. Nguyen, D. Taniar, B.O. Apduhan, O. Gervasi (eds.) Lecture Notes in Computer Science. Proceedings of the 13th International Conference Computational Science and Its Applications - ICCSA 2013, pp. 466-480. Ho Chi Minh City, Vietnam (2013)

[30] Mao, B., Harrie, L., Ban, Y.: Detection and typification of linear structures for dynamic visualization of 3D city models. Computers, Environment and Urban Systems 36(3), 233244 (2012)

[31] Open Geospatial Consortium: OGC City Geography Markup Language (CityGML) Encoding Standard 2.0.0 (2012)

[32] Open Geospatial Consortium: OGC Geography Markup Language (GML) - Extended schemas and encoding rules 3.3.0 (2012) 
[33] Salomon, D.: Data Compression - The Complete Reference, 4 edn. Springer London (2007)

[34] Sony Computer Entertainment Inc.: COLLADA - Digital Asset Schema Release 1.5.0. The Khronos Group (2008)

[35] Stadler, A., Nagel, C., König, G., Kolbe, T.H.: Making Interoperability Persistent: A 3D Geo Database Based on CityGML. In: L. Jiyeong, S. Zlatanova (eds.) 3D Geo-Information Sciences. Lecture Notes in Geoinformation and Cartography, pp. 175-192. Springer Berlin Heidelberg (2009)

[36] Steinhage, V., Behley, J., Meisel, S., Cremers, A.B.: Automated updating and maintenance of 3D city models. In: A. Peled (ed.) ISPRS joint workshop on "Core Spatial Databases Updating, Maintenance and Services - from Theory to Practice", pp. 1-6. Haifa, Israel (2010)

[37] Stoter, J., Visser, T., van Oosterom, P., Quak, W., Bakker, N.: A semantic-rich multi-scale information model for topography. International Journal of Geographical Information Science 25(5), 739-763 (2011)

[38] XML Core Working Group: XML Linking Language (XLink) Version 1.1 (2010). URL http://www.w3.org/TR/xlink11/

[39] Zhang, X., Ai, T., Stoter, J., Zhao, X.: Data matching of building polygons at multiple map scales improved by contextual information and relaxation. ISPRS Journal of Photogrammetry and Remote Sensing 92(C), 147-163 (2014)

[40] Zhao, J., Qingg, Z., Du, Z., Feng, T., Zhang, Y.: Mathematical morphology-based generalization of complex 3D building models incorporating semantic relationships. ISPRS Journal of Photogrammetry and Remote Sensing 68(C), 95-111 (2012)

[41] Zheng, Y., Cohen-Or, D., Averkiou, M., Mitra, N.J.: Recurring part arrangements in shape collections. Computer Graphics Forum 33(2), 115-124 (2014) 